\title{
Impacto da dismenorreia em adolescentes escolares
}

\author{
Impact of dismenorrhea on school adolescents
}

Impacto de la dismenorrea en los adolescentes escolares

Nathália Santos Barbosa da Silva ${ }^{1}$, Nathalia Regina Machado Pereira ${ }^{1}$, Antônio Sávio Inácio³, Raphael Alves da Silva ${ }^{1}$, Erica Marcela Oliveira Silva ${ }^{3}$, Felicialle Pereira da Silva².

\section{RESUMO}

Objetivo: Avaliar o impacto da dismenorreia nas adolescentes e identificar o nível de conhecimento das mesmas sobre o assunto. Métodos: Estudo descritivo, exploratório, transversal com abordagem quantitativa, conduzida em uma escola Estadual de Referência em Ensino Médio de Pernambuco, com uma amostra de 50 estudantes, realizado no período de 30 dias. Resultados: Observou-se que a maioria das adolescentes $(78 \%)$ não possuíam conhecimento em relação aos distúrbios menstruais, o que demostra que ainda existe a necessidade de esclarecimentos por parte das adolescentes, sobre o ciclo menstrual e seus possíveis distúrbios. Quanto ao absenteísmo escolar, 56\% afirmaram já ter faltado aula devido à dor no período menstrual, o que evidenciou o efeito da dismenorreia na vida das adolescentes. Conclusão: Neste sentido, percebe-se que existe a necessidade em valorizar as queixas com relação ao que se refere à menstruação, uma vez que estas podem impactar diretamente na saúde, na qualidade de vida e no desempenho escolar, assim como uma maior efetivação do Programa de Saúde na Escola.

Palavras-chave: Adolescente, Dismenorreia, Conhecimento, Mudança social, Distúrbios menstruais.

\begin{abstract}
Objective: To evaluate the impact of dysmenorrhea in adolescents and to identify the level of knowledge about the subject. Methods: Descriptive, exploratory, cross-sectional study with a quantitative approach, conducted at a State Reference School in Secondary Education in Pernambuco, with a sample of 50 students, carried out over a period of 30 days. Results: It was observed that the majority of adolescents $(78 \%)$ had no knowledge regarding menstrual disorders, which shows that there is still a need for clarification on the part of the adolescents, about the menstrual cycle and its possible disorders. As for school absenteeism, 56\% said they had already missed class due to pain during their menstrual period, which showed the effect of dysmenorrhea on the lives of adolescents. Conclusion: In this sense, it is perceived that there is a need to value complaints regarding menstruation, since these can directly affect health, quality of life and school performance, as well as greater effectiveness ofthe School Health Program.
\end{abstract}

Keywords: Adolescent, Dysmenorrhea, Knowledge, Social change, Menstrual disorders.

\section{RESUMEN}

Objetivo: evaluar el impacto de la dismenorrea en los adolescentes e identificar su nivel de conocimiento sobre el tema. Métodos: Estudio descriptivo, exploratorio, transversal con enfoque cuantitativo, realizado en

${ }^{1}$ Centro Universitário Maurício de Nassau, Recife - PE.

*E-mail: raphaelalves770@hotmail.com

${ }^{2}$ Neuropsiquiatria e Ciências do comportamento - (UFPE), Recife - PE

${ }^{3}$ Programa de Residencia em Neurologia e Neurocirurgia - (UPE), Recife - PE 
una Escuela de Referencia Estatal de Educación Secundaria en Pernambuco, con una muestra de 50 estudiantes, realizado en un período de 30 días. Resultados: se observó que la mayoría de las adolescentes $(78 \%)$ no tenían conocimiento sobre los trastornos menstruales, lo que demuestra que todavía hay una necesidad de aclaración por parte de las adolescentes sobre el ciclo menstrual y sus posibles trastornos. En cuanto al absentismo escolar, el $56 \%$ dijo que ya había faltado a clase debido al dolor durante su período menstrual, lo que mostró el efecto de la dismenorrea en la vida de las adolescentes. Conclusión: en este sentido, se percibe que existe la necesidad de valorar las quejas en relación con la menstruación, ya que pueden afectar directamente la salud, la calidad de vida y el rendimiento escolar, así como una mayor efectividad de la Programa de salud escolar.

Palabras clave: Adolescente, Dismenorrea, Conocimiento, Cambio social, Trastornos menstruales

\section{INTRODUÇÃO}

Segundo a Organização Mundial de Saúde a adolescência é caracterizada no período entre 10 aos 19 anos, compreendendo a fase de transição entre a infância e a idade adulta, definida pelo desenvolvimento físico, mental, emocional, sexual e social (OMS, 1985; RATTI CR, et al., 2015). Caracterizam-se como puberdade os fenômenos biológicos, que proporcionam o crescimento somático dos caracteres sexuais secundários e a capacidade de reprodução. Entre as transformações que existem durante esta etapa, podese destacar a reativação do eixo-hipotalâmico, que resulta na presença dos ciclos menstruais (CUNHA SB, et al., 2015; PENTEADO CFS, 2018).

Dentre tantas transformações no sexo feminino, a menstruação é um dos eventos mais marcantes, evidenciado pelo sangramento vaginal periódico que se inicia na menarca e finaliza-se com a menopausa. Os distúrbios do ciclo menstrual são alterações atípicas do ciclo, e entre os mais frequentes, estão a menorragia, a dismenorreia e os ciclos irregulares (RATTI CR, et al., 2015; JOSEPH G, 2014).

A menorragia ou sangramento uterino anormal se caracteriza pelo fluxo intenso de sangue, já a dismenorreia envolve dor durante o período menstrual, e possui prevalência em cerca de $80 \%$ das adolescentes (FEBRASGO, 2015; GOMES MRA, et al., 2016). Metade dos ciclos menstruais chega a ser anovulatórios nos primeiros dois anos, sendo a síndrome dos ovários policísticos uma das causas mais comuns de anovulação na adolescência (RAMOS ACP, et al., 2014; FEBRASGO, 2015).

A dismenorreia que acompanha os ciclos menstruais é regularmente a queixa mais evidenciada em cerca de $80 \%$ das adolescentes. A mesma pode ser classificada como dismenorreia primária, que é caracterizada como dor ao menstruar em mulheres com anatomia pélvica normal, iniciando-se normalmente na adolescência.

Esta também pode ser influenciada por nuliparidade, menarca antes dos 12 anos e tabagismo, já a dismenorreia secundária, é definida como dor menstrual que se inicia anos após a menarca, e pode estar associada às doenças pélvicas (FRARE JC,et al., 2017;CONSELHO FEDERAL DE FARMACIA, 2017). Particularmente a dor, e outros sintomas que aparecem durante a menstruação, podem atingir 0 desempenho na escola e elevar a taxa de absenteísmo escolar (SILVA JRA, et al., 2017).

Entende-se que, ao mesmo tempo em que a menarca indica que biologicamente o corpo está preparado para reprodução, a menina pode não estar preparada para este evento, que se torna traumático, muitas vezes por não compreender o que se passa com ela. Não menos frequente, evidencia-se a falta de orientação dos pais e da família. Estes deveriam dialogar com naturalidade sobre o processo evolutivo da maturação dos caracteres sexuais secundários, bem como do desenvolvimento do aparelho reprodutor, que deve ser encarado com naturalidade, porém sem subestimar as queixas de dores (FEBRASGO, 2017).

Mães que apresentaram dismenorreia e que não tiveram orientação durante o período da adolescência, tendem geralmente a reproduzir este comportamento fazendo com que a adolescente encare seus sintomas como algo sem solução pela experiência familiar. Este fato faz com que a adolescente creia que precisa conviver com a dor, que a limita interferindo nas suas atividades. Assim, o ciclo se perpetua, se fecha e não 
se dissemina (SPINOLA MCR, et al., 2017). Dessa forma, a família cumpre o papel de principal responsável pela educação sexual dos adolescentes, devendo oferecer espaço e segurança para a adolescente expor suas dúvidas, medos, anseios e queixas (SAVEGNAGO SDO, 2016). A escola neste sentido, poderia se apresentar como um parceiro importante para atuar com estratégias de acolhimento, orientações e intervenções para minimizar esta problemática. A atuação qualificada dos profissionais educadores nesse momento de dúvidas e incertezas, pode influenciar positivamente na forma como a adolescente vivencia o processo do ciclo menstrual. Entretanto, esses profissionais não recebem capacitação acerca do assunto (FURLANETTO MF, 2017).

Diante desta realidade, observa-se que os distúrbios menstruais podem afetar de forma negativa a qualidade de vida das adolescentes tornando-se uma temática de grande relevância, tanto para as adolescentes, quanto para os profissionais de saúde. Neste sentido, o conhecimento desta problemática torna-se imprescindível dentro da atuação dos profissionais de saúde nos processos de promoção, proteção e recuperação da saúde. Portanto, o estudo tem por objetivo avaliar o impacto da dismenorreia nas adolescentes e identificar o nível de conhecimento das adolescentes sobre o assunto.

\section{MÉTODOS}

Trata-se de um estudo descritivo, exploratório, transversal com abordagem quantitativa. Os estudos descritivos são usados para descrever e analisar a realidade, enquanto os transversais são estudos que acompanham a população em um corte temporal. O estudo foi realizado em uma escola Referência do Ensino Médio localizada em Recife- PE, Brasil. Optou-se por este local de estudo por se tratar de um colégio semi-integral de referência dedicada ao ensino médio do $1^{\circ}$ ao $3^{\circ}$ ano.

Foram incluídas as adolescentes do sexo feminino entre 11 a 19 anos, matriculadas e com frequência regular no local de estudo. Foram excluídas as adolescentes gestantes, com problemas de surdez e àquelas com dificuldades em compreender $\mathrm{o}$ instrumento da pesquisa. $\mathrm{O}$ estudo contou com uma amostra não-probabilística, com um total de 50 participantes.

A coleta de dados foi realizada no período de 30 dias e foi entregue um instrumento semiestruturado com 10 questões para autopreenchimento pelas adolescentes. Este instrumento foi construído com base nas diretrizes para distúrbios menstruais de acordo com a Federação Brasileiras das Associações de Ginecologia e Obstetrícia (FEBRASGO, 2015). Foram abordadas as seguintes características do ciclo menstrual: ano da menarca, duração da menstruação, frequência do ciclo menstrual, intensidade do fluxo, ausência de menstruação, presença de dor, intensidade da dor, duração da dor, uso de analgésico, sintomas associados à dor, absenteísmo escolar, interferência nas atividades diárias, uso de métodos contraceptivos e a visita ao ginecologista.

Os dados foram tabulados em uma planilha do programa Microsoft Excel 2010 e para obtenção de cálculos estatísticos utilizou-se do programa Statistical Package for the Social Sciences (SPSS) versão 23 e analisados descritivamente através de frequências absolutas e percentuais para as variáveis categóricas e das medidas: média, desvio padrão e mediana para as variáveis numéricas. Os preceitos éticos emanados pela resolução 466/12 foram respeitados, com a garantia do anonimato mediante assinatura dos Termos de Consentimento Livre e Esclarecido (TCLE) para os pais e o Termo de Assentimento Livre e Esclarecido (TALE) para as participantes. Número do parecer consubstanciado do comité de ética e pesquisa em seres humanos: 2.959.114.

\section{RESULTADOS}

Participaram do estudo 50 alunas com faixa etária entre 13 a 19 anos, com média de 16,62 anos, desvio padrão igual a 1,23 anos e mediana igual a 17 anos. Entre as pesquisadas, 40,0\% tinham entre 13 a 16 anos e as $60,0 \%$ demais tinham 17 a 19 .

A Tabela 1 apresenta informações acerca do conhecimento sobre distúrbios menstruais, regularidade dos ciclos e uso de métodos contraceptivos. Observou-se que $22 \%$ afirmaram ter algum tipo de 
conhecimento sobre distúrbios menstruais, e entre essas, a maioria referiu saber sobre regularidade do ciclo menstrual. A maioria das entrevistadas informou ter tido a primeira menstruação entre 12 e 15 anos. Com relação aos métodos contraceptivos, 20,0\% responderam que utilizam ou já os utilizaram, sendo metade destes, uso de injeção e a outra metade o uso de comprimidos.

Ainda na Tabela 1, observa-se que cerca de $64,0 \%$ das estudantes afirmaram que o intervalo de sua menstruação é mensal. Em relação à frequência da ida ao ginecologista, apenas $26 \%$ afirmaram frequentar ou ter ido a uma consulta, e entre a pesquisadas que já foram ao ginecologista, os motivos mais citados foram cólicas menstruais $(7,0 \%)$ e iniciação sexual $(7,0 \%)$.

Tabela 1 - Questões relacionadas à menstruação, uso de contraceptivos e a frequência de consultas com um ginecologista. Recife-PE, Brasil, 2019.

\begin{tabular}{|c|c|c|}
\hline Variável & $\mathbf{n}$ & $\%$ \\
\hline \multicolumn{3}{|l|}{ Faixa etária } \\
\hline 13 a 16 anos & 20 & 40 \\
\hline 17 a 19 anos & 30 & 60 \\
\hline \multicolumn{3}{|c|}{ Possui algum conhecimento sobre distúrbios menstruais? } \\
\hline Sim & 11 & 22 \\
\hline Não & 39 & 78 \\
\hline \multicolumn{3}{|c|}{ Quais conhecimentos sobre distúrbios menstruais? } \\
\hline Regularidade da menstruação & 6 & 12 \\
\hline Conhecido sobre o ciclo & 1 & 2 \\
\hline Cólica & 4 & 8 \\
\hline Não possui & 39 & 78 \\
\hline \multicolumn{3}{|c|}{ Qual idade você tinha quando teve a $1^{a}$ menstruação? } \\
\hline 8 a 11 anos & 17 & 34 \\
\hline 12 a 15 anos & 33 & 66 \\
\hline \multicolumn{3}{|c|}{ Utiliza ou já utilizou algum método contraceptivo? } \\
\hline Sim & 10 & 20 \\
\hline Não & 40 & 80 \\
\hline \multicolumn{3}{|l|}{ Qual método contraceptivo utilizado? } \\
\hline Injeção & 5 & 10 \\
\hline Comprimido & 5 & 10 \\
\hline Nunca utilizou & 40 & 80 \\
\hline \multicolumn{3}{|l|}{ Qual intervalo da sua menstruação? } \\
\hline Mensal & 32 & 64 \\
\hline Duas vezes no mês & 5 & 10 \\
\hline Mais de uma vez no mês & 7 & 14 \\
\hline Já fiquei sem menstruar & 6 & 12 \\
\hline \multicolumn{3}{|c|}{ Frequenta ou já frequentou o ginecologista? } \\
\hline Sim & 23 & 46 \\
\hline Não & 27 & 54 \\
\hline \multicolumn{3}{|c|}{ Qual motivo da ida ao ginecologista? } \\
\hline Secreção & 4 & 8 \\
\hline Exame & 2 & 4 \\
\hline Atraso & 3 & 6 \\
\hline Cólica & 7 & 14 \\
\hline Iniciação sexual & 7 & 14 \\
\hline Não frequenta & 27 & 54 \\
\hline Total & 50 & $100 \%$ \\
\hline
\end{tabular}

Fonte: Silva NSB, et al., 2019. 
Na Tabela 2 observa-se que entre as pesquisadas, em $66,0 \%$ a menstruação no mês que ocorreu a pesquisa durou em torno de cinco dias e $94 \%$ destas adolescentes relataram sentir cólicas durante a menstruação, com dor que varia entre moderada a máxima. Quando questionadas sobre o uso de analgésicos, $68 \%$ responderam que os utilizam, e que $16 \%$ que fazem o uso ocasionalmente. Os medicamentos mais utilizados foram buscofem e dipirona. Mais da metade das adolescentes informaram já ter faltado aula por causa da menstruação e/ou das cólicas, sendo que $18 \%$ relatam que sempre faltam aulas na presença da menstruação e $38 \%$ faltamàsvezes. Já com relação à realização de suas atividades diárias, 56,0\% respondeu que a menstruação ou cólicas atrapalham ocasionalmente suas atividades diárias e $36,0 \%$ responderam que atrapalham sempre.

Tabela 2 - Avaliação da duração da menstruação, suas consequências devido à cólica e o tipo de analgésico utilizado. Recife-PE, Brasil, 2019.

\begin{tabular}{|c|c|c|}
\hline Variável & $\mathbf{n}$ & $\%$ \\
\hline \multicolumn{3}{|c|}{ Tempo que durou a menstruação (mês que foi feito a pesquisa) } \\
\hline 3 dias & 3 & 6 \\
\hline 5 dias & 33 & 66 \\
\hline Mais de uma semana & 11 & 22 \\
\hline Mais de 15 dias & 1 & 2 \\
\hline Não menstruou & 2 & 4 \\
\hline \multicolumn{3}{|l|}{ Sente cólicas? } \\
\hline Sim & 47 & 94 \\
\hline Não & 3 & 6 \\
\hline \multicolumn{3}{|l|}{ Escala da dor de cólica? } \\
\hline Ausente de dor & 3 & 6 \\
\hline Dor moderada & 30 & 60 \\
\hline Dor máxima & 14 & 28 \\
\hline Não sente cólicas & 3 & 6 \\
\hline \multicolumn{3}{|c|}{ Quando sente cólicas faz uso de analgésicos? } \\
\hline Sim & 34 & 68 \\
\hline Às vezes & 8 & 16 \\
\hline Nunca & 5 & 10 \\
\hline Não sente dor & 3 & 6 \\
\hline \multicolumn{3}{|l|}{ Quais analgésicos que utiliza } \\
\hline Dipirona & 12 & 24 \\
\hline Buscofem & 13 & 26 \\
\hline Ibuprofeno & 3 & 6 \\
\hline Buscopan & 5 & 10 \\
\hline Misiginasol & 1 & 2 \\
\hline Dorflex & 6 & 12 \\
\hline Paracetamol & 2 & 4 \\
\hline Não faz uso/ não sente dor & 8 & 16 \\
\hline \multicolumn{3}{|c|}{ A menstruação ou a cólica faz com que você falte aula? } \\
\hline Sempre & 9 & 18 \\
\hline Às vezes & 19 & 38 \\
\hline Nunca & 22 & 44 \\
\hline \multicolumn{3}{|c|}{ A menstruação ou a cólica atrapalha as suas atividades diárias? } \\
\hline Sempre & 18 & 36 \\
\hline Às vezes & 28 & 56 \\
\hline Nunca & 4 & 8 \\
\hline Total & 50 & 100 \\
\hline
\end{tabular}

Fonte: Silva NSB, et al., 2019. 
No Gráfico 1 estão apresentados os sintomas associados à menstruação, àqueles com mais ocorrência foram: cólicas $90 \%$; dores nas costas $74 \%$; seios doloridos e inchados, dores de cabeça e choro excessivo $70 \%$, ansiedade $68 \%$ e enjoo $58 \%$.

Gráfico 1 - Frequência percentual da presença de sintomas associados à menstruação. Recife-PE, Brasil, 2019.

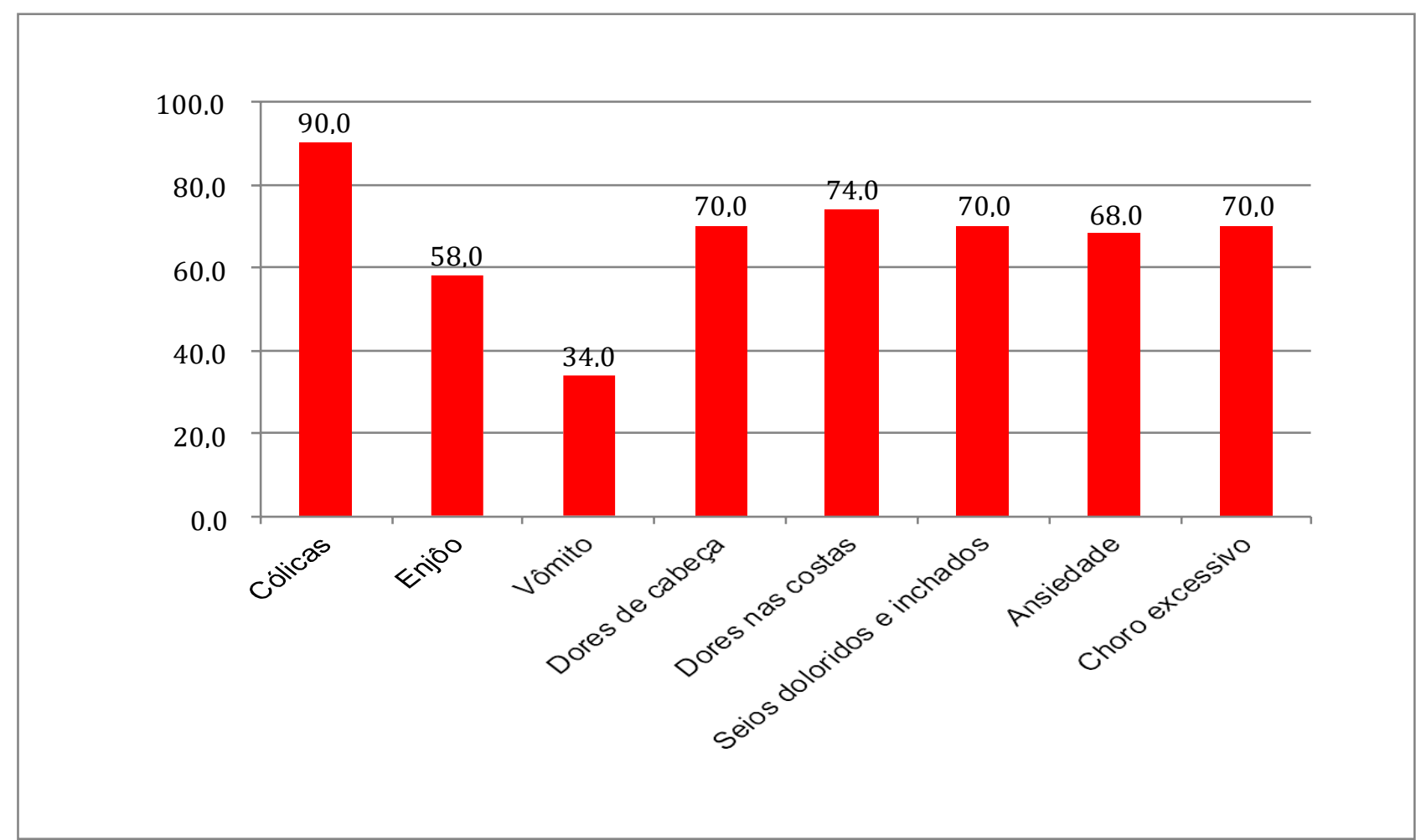

Fonte: Silva NSB, et al., 2019.

\section{DISCUSSÃO}

A idade das participantes do estudo entre 13 a 19 anos refere-se geralmente a faixa etária em fase escolar. Este também é o período em que as adolescentes já iniciaram as mudanças hormonais e fisiológicas da fase puberal, portanto, esta fase também é marcada por dúvidas que envolvem sexualidade e as mudanças visíveis no corpo, além disso, a idade da menarca nas adolescentes pesquisadas foi entre 12 a 15 anos na maioria, o que confirma a mesma faixa para estudos realizados em adolescentes americanas. Dados semelhantes foram encontrados em uma pesquisa realizada em São Paulo, apesar de que outro estudo no Brasil observou variação em outras cidades do país, sendo de 12,1 no Rio de Janeiro, RJ, de 12,4 em Pelotas, RS e de 11,20 em Recife, PE (RAMOS ACP, et al., 2014; FONSECA JSJ, NETO JMMDA, 2017).

Segundo estes estudos a média da idade da menarca no Brasil é entre 11 e 12 anos, sendo importante ressaltar que a menarca, pode ser influenciada pelo clima, nível socioeconômico, fatores genéticos, etnias, e estado nutricional, podendo ainda estar relacionada com a insatisfação da imagem corporal (SPINOLA MCR,et al., 2017; RAMOS ACP,et al., 2014; BARROS BS, et al., 2019). O tempo de duração da menstruação foi estimado em torno de cinco dias, fato que corrobora com alguns autores que relatam que a menstruação normal dura em média de três a oito dias (SILVA FBP et al., 2019; CUNHA SB et al., 2015).

Com relação ao intervalo da menstruação, o ciclo menstrual dura em média 28 dias, entretanto algumas adolescentes referiram menstruar mais de uma vez no mês, o que caracteriza sangramento uterino anormal, em que o intervalo menstrual ocorre com menos de 21 dias no mês, com mais de sete dias de fluxo. As causas podem ser estruturais ou não estruturais, a primeira envolve alterações nas estruturas uterinas, e a 
segunda pode estar relacionadas à coagulopatias, causas ovulatórias, entre outras Todavia, trata-se de uma afecção frequente, que afeta negativamente a qualidade de vida da mulher, incluindo o absenteísmo no trabalho e na escola(PENTEADO CFS, 2018; FEBRASGO, 2017; SILVA AL,et al., 2015).

A dismenorreia relatada neste estudo apresentou prevalência alta entre as participantes, entretanto a dor durante o período menstrual foi referida pela maioria das adolescentes como moderada na escala visual analógica. Esses achados também foram relatados em um estudo transversal realizado em 28 escolas do Japão com uma amostra de 2,819 estudantes, em que 46,8\% descreveram a dismenorreia como grave e moderada e $17,7 \%$ dor severa (KAZAMA M, et al., 2015).

A dismenorreia está diretamente ligada ao aumento das prostaglandinas que causam maior contração intrauterina, causando dores mais intensas. Essa contração excessiva promove a diminuição do fluxo vascular uterino, provocando hipóxia e isquemia, acentuando o quadro doloroso. Além disso, a ação das prostaglandinas estimula constrições na musculatura lisa do estômago, intestino e vasos sanguíneos, podendo resultar em sintomas associados como diarreia, náuseas, vômitos, cefaleia e irritabilidade (ALVES TP, et al., 2016). Sendo assim, configura-se como uma das condições que interfere negativamente na mulher por se tratar de uma queixa com frequência elevada, consequentemente, considerado um problema de saúde pública (SILVA JRA, et al., 2017; HAAMID F, et al., 2017).

O uso de contraceptivos hormonais foi citado como objetivo de prevenção da gravidez, no qual as adolescentes referiram usar comprimidos e injeções. Os contraceptivos hormonais também são geralmente indicados como tratamento para a dismenorreia, atuando pela inibição da ovulação, reduzindo os níveis de prostaglandinas durante a menstruação e redução do fluxo menstrual. A porcentagem de eficácia é avaliada em $65 \%$, apesar de que o uso desses medicamentos estejarelacionado aos efeitos adversos como náusea, vômitos, cefaleia, dor abdominal, ganho de peso e acne (PENTEADO CFS, 2018; ALVES TP, et al., 2016).

A frequência ao ginecologista mostrou-se baixa no presente estudo, mais da metade das adolescentes (54\%) informaram nunca terem frequentado o ginecologista, mostrando a necessidade de orientação médica sobre o uso de medicamentos que contenham hormônios contraceptivos, que não raramente são considerados de primeira opção quando a adolescente tem vida sexual ativa, ou estiver preste a inicia-la, devido sua dupla ação. Em outros casos, os analgésicos e os anti-inflamatórios não esteroides seriam os mais indicados (RAMOS ACP, et al., 2014; ALVES TP, et al., 2016).

Em uma pesquisa realizada em São Paulo (SP) foram observados que os principais motivos de consulta entre as adolescentes foram: acompanhamento de rotina $(30 \%)$, seguido pela orientação sobre anticoncepcionais $(27,5 \%)$ e dismenorreia (17,5\%). Outro estudo realizado pela Universidade de Franca em $\mathrm{SP}$ relatou que as queixas mais incidentes em adolescentes foram alterações no ciclo menstrual e dor pélvica(CINTRA KA,et al., 2019). Quanto ao tempo de consulta, grande parte das entrevistadas (55\%) relatou que a consulta durou menos que 15 minutos, e apenas metade (50\%) das adolescentes disseram ter tido suas dúvidas esclarecidas (RAMOS ACP, et al., 2014).

De acordo com a Federação Brasileira das Associações de Ginecologia e obstetrícia (FEBRASGO), embora as queixas clínicas das adolescentes sejam do conhecimento e manejo do ginecologista, a dificuldade maior é estabelecer o vínculo de confiança com a adolescente, uma vez que na maioria das vezes a adolescente que se encontra na consulta nem sempre veio por inciativa própria, o que tira a sua privacidade por estar acompanhada.Logo, o exame ginecológico é um cuidado que deve ser realizado por um profissional que considere os aspectos da adolescência para que a adolescente possa iniciar uma rotina de prevenção e atenção à sua saúde sexual e reprodutiva (FEBRASGO, 2017).

Porém, existem obstáculos, tais como: dificuldade para a marcação de consultas, impossibilidade de escolha do profissional e atendimento inadequado para as especificidades dessa faixa etária, o que causa certa rejeição à consulta ginecológica. Um estudo realizado no Rio Grande do Norte relacionou aspectos que dificultam o acesso da adolescente para o acompanhamento médico ginecológico, esses associam medo, vergonha e constrangimento. Outra dificuldade também relatada diz respeito às exigências burocráticas, tanto no serviço público, como no privado (GOMES VLO, et al., 2014; RAMOS ACP, et al., 2014). 
O absenteísmo escolar na presente pesquisa se apresentou de forma majoritária nas participantes, corroborando com estudo realizado na Tbilisi (Geórgia). Outra pesquisa realizada nos Estados Unidos da América com 60 adolescentes mostrou que $40 \%$ faltavam aula por desconforto com a menstruação. Grande parte das adolescentes que relataram dismenorreia também afirmou que os sintomas atrapalham as suas atividades de vida diária, bem como as impedem de ir para escola. Desta forma, a dismenorreia apresenta um impacto negativo nas ações de produtividade e absenteísmo escolar. (DE SANCTIS V, et al., 2015; SAHIN N, et al., 2018).

Com relação aos sintomas associados à menstruação, outras queixas foram correlacionadas, tais como: lombalgia, cefaleia e náuseas. Estes sintomas são resultados da ação das prostaglandinas sobre os vasos sanguíneos, estômago e intestino (ALVES TP, et al., 2016). O resultado desta pesquisa evidenciou que entre a maioria havia o desconhecimento sobre ciclo menstrual, estando o pouco entendimento relatado associado aos sintomas de dores e regularidade dos ciclos.

Percebe-se neste sentido que apesar de existir a disciplina de biologia na escola, que também aborda sobre o funcionamento do corpo humano, ainda existe a necessidade de conhecimentos fisiológicos sobre o crescimento e maturação do aparelho reprodutor e de educação sexual. O presente estudo evidenciou que a maioria das entrevistadas não possuía conhecimento sobre distúrbios menstruais, embora esteja evidenciado que o ambiente escolar neste sentido poderia se configurar como um espaço propício para que as adolescentes possam se autoconhecer, uma vez que a educação em saúde consiste em ferramenta imprescindível para induzir mudanças positivas no comportamento do individuo em relação à saúde (MARTINS NRM, NETO JM, 2014; JOSEPH G, 2014).

Com base nesta realidade, existem programas ministeriais que foram planejados para esta finalidade, como o Programa de Saúde nas Escolas (PSE). Este programa considera a escola como um espaço de relações ideais para o desenvolvimento de práticas educativas integradas. Neste sentido, as diretrizes propostas se dispõem ao fortalecimento e resolutividade entre escolas e unidades de saúde. Portanto, a linha de ação sobre saúde reprodutiva deve incluir os aspectos relacionados ao aparelho reprodutor, uma vez que se deve considerar que as afecções possam existir, e, ao mesmo tempo, passarem despercebidas pela falta de conhecimento ou de informação pelas adolescentes (BRASIL, 2015). Nesta perspectiva, a interlocução saúde-escola também deve incluir os familiares, devendo ocorrer de forma sistemática, longitudinal, que utilizem estratégias problematizadoras, de preferência em formato de oficinas para orientar as adolescentes proporcionando um ambiente para que se sintam confortáveis em conversar sobre o seu corpo (FURLANETTO MF,et al., 2017).

As vivencias em salas de aula parecem ser limitadas, talvez por abordar o conteúdo de forma teórica e simplista, que de certa forma pode causar inquietação, ou não ser suficiente para esclarecer dúvidas, gerando tensão e ansiedade entre as adolescentes (MARTINS NRM, NETO JM, 2014). Além disso, podem sentir timidez em expor suas dúvidas, ou em relatar queixas relacionadas à menstruação. Assim, a forma de abordagem deve ser construída pelos educadores e profissionais de saúde considerando os fatores socioculturais das adolescentes, com metodologias direcionadas para proporcionar um melhor preparo psicológico, considerando-se esses aspectos efetivos na prevenção da dismenorreia primaria (REIS AM, et al., 2016).

Este estudo apresenta limitações importantes por não ter avaliado outras variáveis, tais como o diagnóstico nutricional e questionamentos mais aprofundados para investigar outros fatores socioeconômicos e clínicos da amostra. As evidências das respostas podem se apresentar limitadas devido ao autopreenchimento. Portanto, recomenda-se que outras pesquisas sejam implementadas para 0 aprofundamento desta temática.

\section{CONCLUSÃO}

A prevalência da dismenorreia em adolescentes escolares identificada neste estudo é uma realidade que não difere de outras pesquisas nacionais e internacionais. Contudo, estudos envolvendo a temática da dismenorreia no período da adolescência são incipientes, verificando-se lacunas no âmbito da promoção da 
saúde para este público. Neste sentido, percebe-se a necessidade de um espaço de interlocução entre saúde a escola, que priorize as queixas das adolescentes com relação à menstruação, uma vez que estas podem impactar diretamente na saúde, na qualidade de vida e no desempenho escolar. Portanto, avaliações mais rigorosas devem ser aprofundadas, com vistas a dar maior visibilidade à problemática da dismenorreia em adolescentes.

\section{REFERÊNCIAS}

1. ALVES TP, et al. Dismenorreia: diagnostico e tratamento. Revista Científica FAEMA. 2016; 2(1): 1-12.

2. BARROS BS, et al. ERICA: age atmenarche and its association with nutritional status. Jornal de pediatria. 2019; 95(1): 106-111.

3. BRASIL, Ministério da Saúde. Caderno do gestor do PSE. Ministério da Saúde, Ministério da Educação, Brasilia/DF. 2015; p. 68.

4. CINTRA KA, et al. Análise das principais queixas ginecológicas no ambulatório escola da Universidade de Franca e correlação com dados epidemiológicos. Revista Eletrônica Acervo Saúde. 2019; 11(9): e368.

5. CONSELHO FEDERAL DE FARMÁCIA. Guia de prática clínica: sinais e sintomas do aparelho genital feminino: Dismenorreia. Brasilia: Conselho Federal de Farmácia. 2017; p. 218.

6. CUNHA SB, et al. Síndrome pré-menstrual em adolescentes: prevalência, sintomas e impacto nas atividades da vida cotidiana. Revista Adolescência e Saúde. 2015; 12(4): 45-55.

7. CUNHA SGAP, et al. A relação entre a Idade da Menarca e o Índice de Massa Corpórea em adolescentes. UNILUS Ensino e Pesquisa. 2017; 14(35): 139-146.

8. DE SANCTIS V, et al. Primary Dysmenorrhea in Adolescents: Prevalence, Impact and Recent Knowledge. Dismenorréia, Revista Pediatrica de Endocrinologia. 2015; 13(2): 465-73.

9. FEBRASGO, Federação Brasileira das Associações de Ginecologia e Obstetrícia. Atendendo a adolescente no consultório. Série Orientações e Recomendações, São Paulo, ed. 4, 2017.

10. FEBRASGO. Federação Brasileira das Associações de Ginecologia e Obstetrícia. Manual de ginecologia endócrina. São Paulo: Faculdade de Medicina da Universidade de São Paulo, 2015.

11. FEBRASGO. Federação Brasileira das Associações de Ginecologia e Obstetrícia. Sangramento uterino anormal. São Paulo: Faculdade de Medicina da Universidade de São Paulo, 2017.

12. FONSECA JRSJ e NETO JMMD. A menarca em atletas adolescentes brasileiras. Revista Saúde Física e Mental. 2017; 5(1): 1-8.

13. FURLANETTO MF, et al. Educação sexual em escolas brasileiras: revisão sistemática da literatura. Caderno de Pesquisa, [S. I.]. 2018; 48(168): 550-571.

14. FRARE JC, et al. Dismenorreia: Prevalência e efeito na qualidade de vida. Revista de atenção à saúde. 2014; 12(39): 15-20.

15. GOMES MRA, et al. Prevalência de Dismenorreia e sua associação com depressão e ansiedade entre adolescentes de uma escola pública. Revista Adolescente e Saúde. 2016; 13(2): 98-105.

16. GOMES VLO, et al. Representações de adolescentes acerca da consulta ginecológica. Revista da Escola de Enfermagem da USP. 2014; 48(3): 438-445.

17. HAAMID F, et al. Heavy Menstrual Blending in Adolescent .Journal of Pediatric and Adolescent Gynecology. 2017; 30(6): 665.

18. JOSEPH, G. Idade da menarca e sibilância na coorte de nascidos vivos em pelotas. 2014. Dissertação de Mestrado (Pós Graduação de epidemiologia) - Universidade Federal de Pelotas, Pelotas, 2014.

19. KAZAMA M, et al. Prevalence of Dysmenorrhea and Its Correlating Lifestyle Factors in Japanese Female Junior High School Students. TohokuUniversity Medical Press. 2015; 236(2): 107-113.

20. KURAL M, et al. Menstrual characteristics and prevalence of dysmenorrhea in collegegoing girls. Journal of Family Medicine Primary Care. Índia. 2015; 4(3): 426.

21. MARTINS NRM e NETO JM. Adolescente, esse Ser em Transformação. Cadernos PDE, Londrina v. 1, 2014.

22. OMS/OPAS. La Saluddel Adolescente y elJoven em las Américas, D.C., 1985.

23. PENTEADO CFS. Influência do ciclo menstrual e do uso de contraceptivos orais no desempenho aeróbio de corredoras. 2018. Dissertação (Mestrado em Ciências Biomédicas) - Universidade Estadual de Ponta Grossa, Ponta Grossa, 2018.

24. RAMOS ACP, et al. Consulta ginecológica sob a ótica de adolescentes. Revista de Enfermagem do Centro Oeste Mineiro. 2014; 04(03).

25. RATTI CR, et al. O Tabu da Menstruação Reforçado pelas Propagandas de Absorvente. Intercom - Sociedade Brasileira de Estudos Interdisciplinares da Comunicação. 2015; 7(9).

26. REIS AM, et al. A Importância Da Fisioterapia No Tratamento Da Dismenorreia Primária: Estudo Comparativo. Centro Universitário Católico Salesiano. São Paulo, 2016.

27. SAHIN N, et al. Assessment of anxiety-depression levels and perceptions of quality of life in adolescents with dysmenorrhea. Reproductive health. 2018; 15(1): 13.

28. SAVEGNAGO SDO, ARPINI DM. A abordagem do tema sexualidade no contexto familiar: o ponto de vista de mães adolescentes. Psicologia, Ciência e Profissão. 2016; 36(1): 130-144. 
29. SILVA AL, et al. Sangramento uterino anormal: proposta de abordagem do Grupo Heavy Menstrual Bleeding: Evidence-Based Learning for Best Practice (HELP). Femina. 2015; 43(4).

30. SILVA FBP, et al. Prevalência Da Dismenorreia E Sua Influência Na Vida De Trabalhadoras Brasileiras. Revista Saúde e Desenvolvimento. 2019; 13(14): 64-82.

31. SILVA JRA, et al Exercício físico como terapia não farmacológica no tratamento da dismenorreia primária: uma revisão sistemática. Revista pesquisa em fisioterapia. 2017; 7(4): 470-480.

32. SPINOLA MCR, et al. Fatores Associados a Iniciação Sexual em Mães de 14 a 16 anos em Porto Alegre/ RS, Brasil. Ciência \& Saúde Coletiva. 2017; 22: 3755-3762. 